\title{
Precessing accretion discs in binary systems
}

\author{
Alon Retter ${ }^{1}$, Alexander $\mathrm{Liu}^{2}$, Marc Bos ${ }^{3}$ \\ ${ }^{1}$ School of Physics, University of Sydney, NSW 2006, Australia \\ ${ }^{2}$ Norcape Observatory, PO Box 300, Exmouth, 6707, Australia \\ ${ }^{3}$ Mt Molehill Observatory, 83a Hutton Street, Otahuhu, Auckland, New \\ Zealand
}

\begin{abstract}
Superhumps - quasi-periodic oscillations at periods a few percent different than the orbital periods - have been observed in several dozen binary systems of various types. It is well-accepted now that they are caused by the precession of the accretion disc. New findings on TV Col, TX Col and V4742 Sgr are presented. These cataclysmic variables (CVs) have exceptional precessing periods that cast strong doubt on the predictions of the Tidal Disc Instability model.
\end{abstract}

\section{Introduction}

Binary systems often show quasi-periodicities a few percent longer than their orbital periods. These are understood as the beat periods between the orbital period and the apsidal precession of the accretion disc. For historical reasons they are known as positive superhumps. The positive superhumps obey a nice relation between the superhump period excess over the orbital period and the orbital period (Stolz \& Schoembs 1984; Patterson 1999). Negative superhumps, quasi-periodicities a few percent shorter than the orbital periods, are explained by the beat periods between the orbital period and the nodal precession of the accretion disc. The negative superhumps follow a somewhat similar relation between the superhump period deficit over the orbital period and the orbital period (Patterson 1999). Superhumps are important as the binary mass ratio can be estimated from the observed difference between the superhump and orbital periods.

According to theory (Whitehurst \& King 1991; Murray 2000) precessing accretion discs can occur only in binaries with small mass ratios $\left(\mathrm{q}=M_{2} / M_{1} \leq 1 / 3\right)$. In CVs, systems with longer orbital periods have larger separations and their secondaries are thus more massive since they have to fill their Roche Lobes. There is a small scatter on the mass of the primary white dwarf and, therefore, the limit on the mass ratio is translated into orbital periods shorter than about 3-4 $\mathrm{h}$. TV Col, with an orbital period of $5.5 \mathrm{~h}$ and a negative superhump of $5.2 \mathrm{~h}$ has been an unusual case. Retter et al. (2003) found another period, $6.4 \mathrm{~h}$, in existing data of this object and confirmed it by further observations. It is naturally understood as a positive superhump. These results raised the question whether TV Col is unique. This work shows that it is not. 


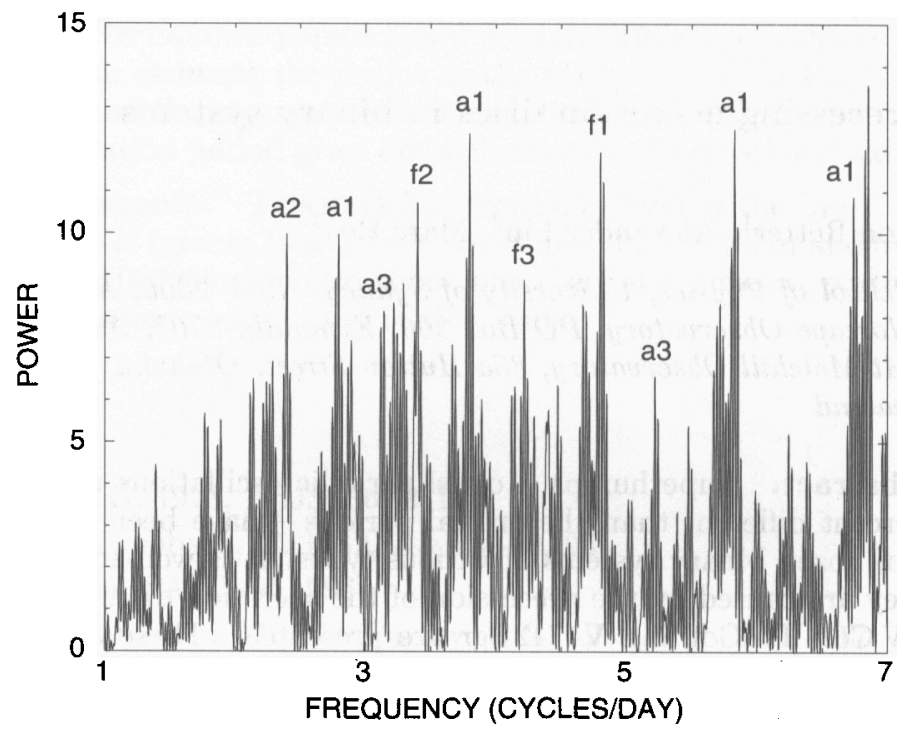

Figure 1. Power spectra of 22 nights of TX Col in $2002-2003 . \mathrm{f}_{3}$ is the known orbital period $(5.7 \mathrm{~h})$. There are two additional groups of peaks centered around $f_{1}(5.2 \mathrm{~h})$ and $\mathrm{f}_{2}(7.0 \mathrm{~h})$ which we interpret as negative and positive superhumps correspondingly.

\section{Observations and Results}

Photometric unfiltered CCD observations of TX Col, a 5.7-h orbital period CV, were carried out during 22 nights in 2002 December to 2003 February. Fig. 1 shows the power spectrum of these data. It shows two groups of peaks in addition to the orbital period $\left(f_{3}\right)$. We propose that the 5.2 -h period $\left(f_{1}\right)$ is a negative superhump and the 7.0-h period $\left(f_{2}\right)$ - a positive superhump. ' $a_{i}$ ' $(i=1-3)$ represent $1-\mathrm{d}^{-1}$ aliases of ' $f_{i}$ ' correspondingly.

Fig. 2 presents the power spectrum of 12 nights on another CV - Nova V4742 Sgr 2002/2 taken during 2003 May-July. It also displays a complicated multi-periodic structure. We tentatively interpret the 6.1 -h peak as the orbital period and the 5.7-h peak as a negative superhump. In new data, there is also evidence for a third group of peaks centered around $6.9 \mathrm{~h}$, which would suit a positive superhump. periods.

Fig. 3 shows the extension of the relation for positive superhumps to long

\section{Conclusions}

It appears that TV Col is not unique and that superhumps could be very common in systems with large orbital periods. These results raise strong doubts on the theoretical models that predict that superhumps should only occur in CVs with 


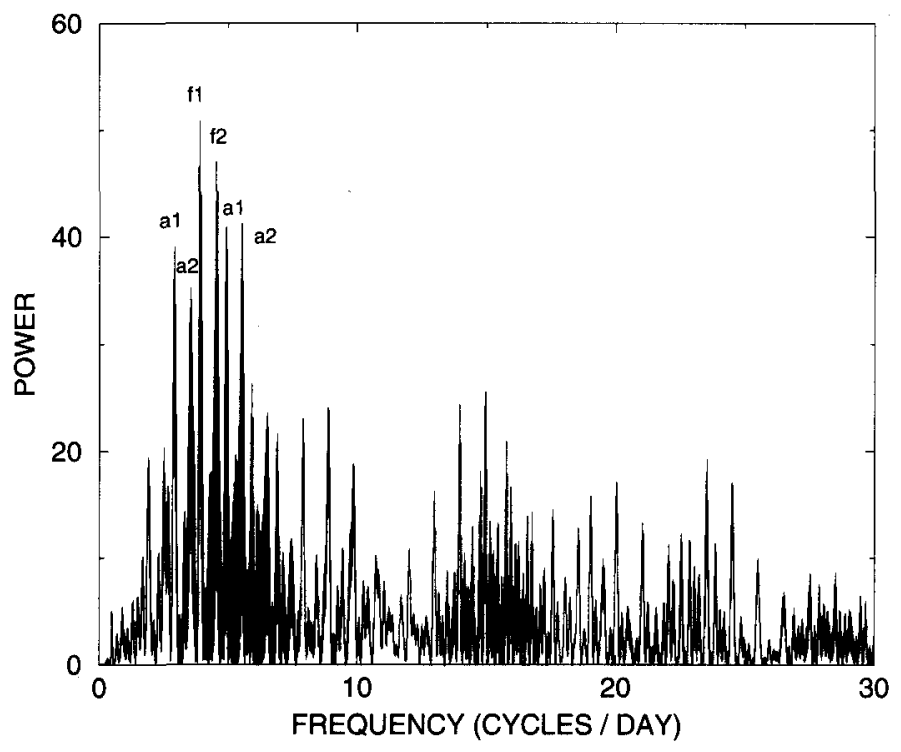

Figure 2. Preliminary results on Nova V4742 Sgr 2002/2. The power spectrum shows at least two long-term periods. We tentatively suggest that the orbital period is $6.1 \mathrm{~h}$ and that there is a negative superhump at $5.7 \mathrm{~h}$. In addition, the peaks at longer frequencies (shorter periods) support an intermediate polar model for this system.

orbital periods shorter than about $3-4 \mathrm{~h}$ (or mass ratios smaller than $1 / 3$ ). Note that all three systems mentioned in this work are intermediate polars (TX Col and TV Col have been classified as intermediate polar, while the power spectrum of V4742 Sgr (Fig. 2) suggests that it belongs to this subclass as well), which are CVs with a moderate magnetic field of the white dwarf. Large superhumps may be restricted to this subgroup of CVs.

\section{References}

Murray, J.R. 2000, MNRAS, 314, L1

Patterson, J. 1999, in Disk Instabilities in Close Binary Systems, eds S. Mineshige \& C. Wheeler (Universal Academy Press, Tokyo), 61

Retter, A., Hellier, C., Augusteijn, T., Naylor, T., Bedding, T., Bembrick, C., McCormick, J., Velthuis, F. 2003, MNRAS, 340, 679

Stolz, B., Schoembs R. 1984, A\&A, 132, 187

Whitehurst, R., King, A. 1991, MNRAS, 249, 25 


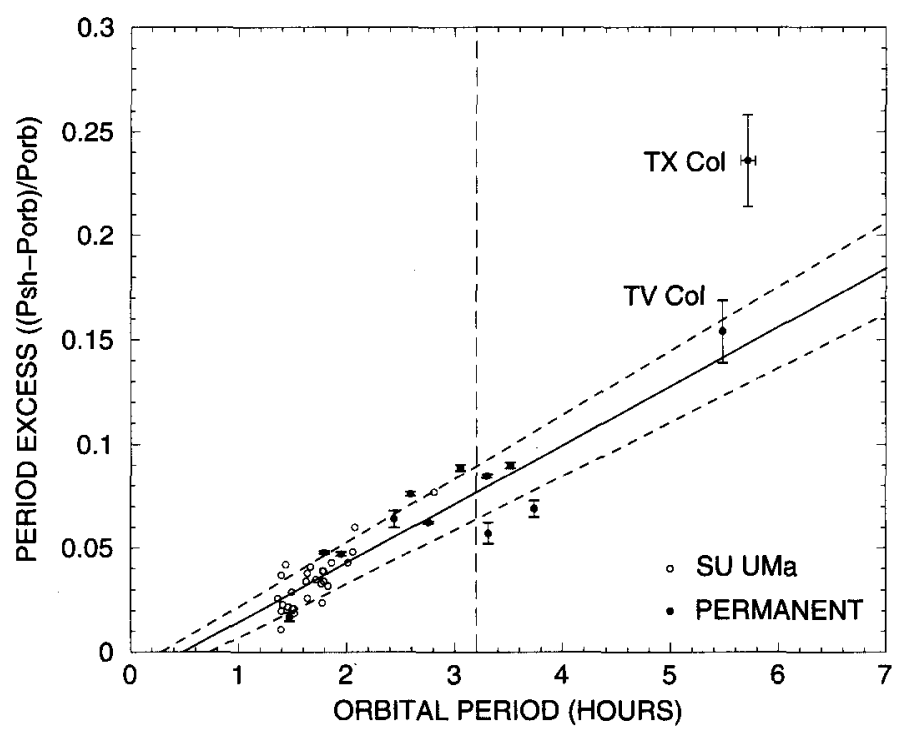

Figure 3. The relation between the positive superhump excess and the orbital period. TV Col obeys the relation while TX Col somewhat deviates from it.

\section{Discussion}

Guinan: What is the physical mechanism for superhumps and especially negative superhumps?

Retter: The negative (or positive) superhump period is the beat period between the orbital period and the nodal (apsidal) precession period of the accretion disc. 